\title{
Novel Adenosine Analog, N6-(4-Hydroxybenzyl)-Adenosine, Dampens Alcohol Drinking and Seeking Behaviors
}

\author{
Sa-lk Hong, ${ }^{1}$ Lee Peyton, ${ }^{1}$ Yijuang Chern, and Doo-Sup Choi \\ Department of Molecular Pharmacology and Experimental Therapeutics (S.-I.H., L.P., D.-S.C.), Neuroscience Program (D.-S.C.), \\ and Department of Psychiatry and Psychology (D.-S.C.), Mayo Clinic College of Medicine, Rochester, Minnesota; and Institute of \\ Biomedical Sciences, Academia Sinica, Taipei, Taiwan (Y.C.)
}

Received July 15, 2019; accepted August 9, 2019

\begin{abstract}
Adenosine signaling is associated with ethanol-related behaviors. We previously found that adenosine $A_{2 A}$ receptor $\left(A_{2 A} R\right)$ activation dampens ethanol drinking behaviors in equilibrative nucleoside transporter 1 (ENT1) knockout mice, and $A_{2 A} R$ inhibition augments reward-seeking behavior in wild-type mice. The novel adenosine analog N6-(4-hydroxybenzyl)-adenosine (NHBA), which is isolated from the rhizomes of Gastrodia elata, activates $A_{2 A} R$ and inhibits ENT1. Here, we examined the effects of NHBA on ethanol drinking in the two-bottle choice test and operant ethanol seeking behaviors. We selected mice exhibiting high ethanol drinking behavior in the two-bottle choice test. NHBA $(0.1 \mathrm{mg} / \mathrm{kg}$, i.p.) reduced ethanol drinking behavior in a limited-access 3-hour drinking session in highconsumption ethanol drinking mice, and NHBA $(0.1 \mathrm{mg} / \mathrm{kg}$, i.p. $)$ did not alter locomotor activity in the open-field test. Operant conditioning with $10 \%$ ethanol and $10 \%$ sucrose (10E10S)
\end{abstract}

reward increased zone entries and time spent in the ethanol zone, while NHBA $(0.1 \mathrm{mg} / \mathrm{kg}$, i.p.) dampened ethanol zone preference in the Y-maze. Furthermore, NHBA $(0.1 \mathrm{mg} / \mathrm{kg}$, i.p.) devalued $10 \mathrm{E} 10 \mathrm{~S}$ and $10 \%$ ethanol (10E) reward after operant conditioning with $10 \mathrm{E} 10 \mathrm{~S}$ and $10 \mathrm{E}$. Taken together, NHBA through $A_{2 A} R$ activation and ENT1 modulation may dampen ethanol drinking and seeking behaviors, suggesting that NHBA is a potential therapeutic agent for treating alcohol use disorder.

\section{SIGNIFICANCE STATEMENT}

Our work highlights that $A_{2 A} R$ activation and ENT1 inhibition by a novel adenosine analog isolated from Gastrodia elata, N6-(4hydroxybenzyl)-adenosine, decreases ethanol drinking and seeking behaviors. We suggest that NHBA is a potential therapeutic agent to treat alcohol use disorder.

\section{Introduction}

Hazardous, harmful, and addictive misuse of alcohol is a highly prevalent psychiatric disease. Over 75 million people around the world have a diagnosable alcohol use disorder (AUD) (Jorgensen et al., 2011). Despite the prevalence of AUD, only limited treatments have been approved by the U.S. Food and Drug Administration (FDA)—such as disulfiram, naltrexone, and acamprosate-for those afflicted with this mental health disorder (Kranzler and Soyka, 2018). Additionally, alternative treatment strategies target the balance between excitatory and inhibitory neurotransmission to reduce ethanol drinking (De Witte et al., 2005; Wackernah et al., 2014). Considering the complex mechanisms underlying AUD and the limitations of the current FDA-approved medications, it has even been suggested that repurposing tetracycline or other medications might be effective in reducing alcohol

This work was supported by the Samuel C. Johnson for Genomics of Addiction Program at Mayo Clinic, the Ulm Foundation, the Godby Foundation, and National Institutes of Health National Institute on Alcohol Abuse and Alcoholism (Grant 5R01AA018779).

${ }^{1}$ S.-I.H. and L.P. contributed equally to this work.

https://doi.org/10.1124/jpet.119.261529. consumption (Oliveros and Choi, 2017). Due to the sparsely efficacious FDA-approved medication to treat AUD, the pursuit to find novel targets to treat AUD is a high priority.

Evidence points to adenosine as a promising target and important inhibitory neurotransmitter strongly contributing to the pathophysiology of many psychiatric conditions, including AUD (Choi et al., 2004; Ruby et al., 2010; Nam et al., 2013a). In the central nervous system, adenosine concentrations are highest in the striatum, where it plays an important role in regulating neural activity and modulating the signaling of various other neurotransmitter systems (Ruby et al., 2010).

Unlike classic neurotransmission in which vesicular release is mediated by electrical impulse, adenosine is released via bidirectional equilibrative nucleoside transporters (ENT) (Sachdeva and Gupta, 2013). Among the four encoded ENT (ENT1-4) isoforms, ENT1 is highly expressed in the striatum and is one of its main transporters for regulating adenosine levels (Nam et al., 2013a; Kao et al., 2017). Interestingly, adenosine has been found to mediate various ethanol-related behaviors (Nagy et al., 1990; Choi et al., 2004; Nam et al., 2013b). In the striatum, adenosine exerts its activity primarily at the Gs $\alpha$-coupled adenosine $A_{2 A} R$ receptor $\left(A_{2 A} R\right)$, which is

ABBREVIATIONS: $\mathrm{A}_{2 \mathrm{~A}} \mathrm{R}$, adenosine $\mathrm{A}_{2 \mathrm{~A}} \mathrm{R}$ receptor; $\mathrm{AUD}$, alcohol use disorder; $10 \mathrm{E}, 10 \%$ ethanol; $10 \mathrm{E} 10 \mathrm{~S}, 10 \%$ ethanol and $10 \%$ sucrose; $\mathrm{ENT}$, equilibrative nucleoside transporter; FDA, U.S. Food and Drug Administration; FR, fixed ratio; KO, knockout; NHBA, N6-(4-hydroxybenzyl)adenosine; RI, random interval; VEH, vehicle; WT, wild type. 
expressed exclusively on striatopallidal neurons, thereby regulating inhibitory behavior control through the indirect pathway of basal ganglia circuitry in drug reward processes (Nam et al., 2013b; Li et al., 2016; Ferre et al., 2018). Accumulating evidence indicates a prominent role of striatal$\mathrm{A}_{2 \mathrm{~A}} \mathrm{R}$ receptors in mediating the cellular and behavioral responses underlying ethanol consumption and ethanol seeking behaviors.

NHBA [N6-(4-hydroxybenzyl) adenosine] is an adenosine analog isolated from the rhizomes of Gastrodia elata, an herb (Huang et al., 2011) that has been used medicinally for over 1500 years in Asia as a treatment of both neurologic and neuropsychiatric diseases, including neurodegenerative diseases, insomnia, anxiety, and depression (Jung et al., 2006; Zhang et al., 2012; Chen et al., 2016). Recently, it has been elucidated that NHBA dually and selectively binds to $\mathrm{A}_{2 \mathrm{~A}} \mathrm{R}$ and ENT1 (Huang et al., 2011). In addition, NHBA has been reported to have beneficial effects for neurologic disorders, such as Huntington's disease, ischemia, and sleep regulation (Huang et al., 2007, 2011; Zhang et al., 2012). However, there are not reports that NHBA affects aspects of AUD.

We investigated whether this novel adenosine analog NHBA regulates ethanol seeking behaviors as a selective $\mathrm{A}_{2 \mathrm{~A}} \mathrm{R}$ agonist and ENT1 modulator. In operant conditioning with either ethanol-containing or ethanol reward, we sought to identify whether NHBA regulates ethanol seeking behaviors. Using the Y-maze, we examined whether NHBA reduces operant training-potentiated ethanol preference. Further, we investigated the effects of NHBA on ethanol drinking among ethanol preferring mice. Our study reveals the pharmacologic effects of the novel adenosine analog NHBA on ethanol seeking and drinking behaviors.

\section{Materials and Methods}

\section{Animals}

Male C57BL/6J mice $(n=140)$ were purchased from Jackson Laboratory (Bar Harbor, ME). Mice were housed in standard Plexiglas cages. The colony room was maintained at a constant temperature $\left(24 \pm 1^{\circ} \mathrm{C}\right)$ and humidity $(60 \% \pm 2 \%)$ under a 12 -hour light/dark cycle with lights on at 06:00 and lights off at 18:00. Mice between 8 and 10 weeks old were used for all experiments.

For the two-bottle choice and locomotor activity tests, the mice were allowed ad libitum access to food and water. For the reward-seeking behavior and three-arm choice tests, the mice were food restricted to $85 \%$ of their baseline weight, at which they were maintained for the duration of experimental procedures. All experimental procedures were approved by the Mayo Clinic Institutional Animal Care and Use Committee and performed in accordance with National Institutes of Health guidelines.

\section{Drugs}

N6-(4-hydroxybenzyl) adenosine riboside (NHBA) was provided by Dr. Yijuang Chern (Institute of Biomedical Sciences, Academia Sinica, Taipei, Taiwan). NHBA was dissolved in the vehicle (VEH): $5 \%$ DMSO, $5 \%$ Tween-80, and $0.9 \%$ saline. Ethanol was mixed with tap water.

\section{Two-Bottle Choice Test}

Oral ethanol consumption and preference were examined using a two-bottle choice test in the mouse home cage. Mice were individually housed. Mice were given 24-hour access to two bottles: water and ethanol. The concentration of ethanol was raised from $3 \%$, to $6 \%$, to $10 \%$ ethanol $(10 \mathrm{E}, \mathrm{v} / \mathrm{v})$ on every 4 th day to adapt ethanol intake. Every other day, we changed the bottle placement to avoid place preference. After increasing the ethanol concentration to $10 \mathrm{E}$, the mice had 18 days of $10 \mathrm{E}$ access. During the last 6 days of $10 \mathrm{E}$ drinking, the average ethanol consumption and preference in all mice were $10.55 \pm 0.96 \mathrm{~g} / \mathrm{kg}$ per 24 -hour period and $75.99 \% \pm 4.17 \%$, respectively.

We then culled the high drinkers: both ethanol consumption $>10 \mathrm{~g} / \mathrm{kg}$ per 24 -hour period and preference $>60 \%$, indicating high drinking behavior (Juarez et al., 2017). For the excluded low drinkers, the average ethanol consumption was $6.24 \pm 0.64 \mathrm{~g} / \mathrm{kg}$ per 24-hour period and ethanol preference was $48.65 \% \pm 4.95 \%$.

One day after selecting mice displaying high ethanol drinking behavior (23 of total 38 mice), their cages were changed to the BioDAQ liquid choice monitoring system (BioDAQ; Research Diets, New Brunswick, NJ) with water and ethanol bottles to minimize liquid spillage. For habituation to the BioDAQ cages, the mice spent an additional 7 days before any monitoring of ethanol consumption or preference was conducted. After the habituation period, we measured $10 \%$ ethanol consumption and preference in the BioDAQ cages to confirm whether mice showing high consumption and preference in standard home cages displayed the same ethanol drinking phenotype in the BioDAQ liquid choice monitoring system.

For the NHBA treatment experiment, we used a total of 13 high ethanol drinking mice by using the BioDAQ drinking cages. Beginning the following day, we measured drinking consumption and preference for 4 hours after either vehicle or NHBA $(0.1 \mathrm{mg} / \mathrm{kg}$, i.p. $)$ was administered at 17:00 hours. Ethanol and water consumption were normalized for evaporation as follows (Nam et al., 2013b): the total volume of the liquid evaporated (water or ethanol) was calculated by averaging 2-day of evaporation from the four control cages without mice. Then, the volume of water or ethanol that evaporated was subtracted from the total consumption of water or ethanol of each mouse. Ethanol consumption (gram per kilogram per 24 hours) was calculated to measure the accurate ethanol amount consumed by each mouse. Ethanol preference (\%) was calculated by dividing the total ethanol solution consumption by the total fluid (ethanol plus water) consumption times 100 at each ethanol concentration.

\section{Reward-Seeking Behavior Test}

We used the same operant chambers as the apparatus in our previous study (Nam et al., 2013b).

Operant Conditioning with Ethanol-Containing Reward. Mice were placed in operant chambers with two interactive nose holes ("active" and "inactive") (Med-Associates, St. Albans, VT) where they poke a single hole for an ethanol-containing reward $(10 \mu \mathrm{l}$ of $10 \%$ ethanol and $10 \%$ sucrose in tap water per reinforcer, 10E10S). We employed the same schedule for operant conditioning used in our previous research (Hong et al., 2019). Briefly, on the 1st day of magazine training, mice were trained to approach the magazine on a random time schedule with a reward delivered for 30 minutes. Next, the mice were trained on a fixed ratio 1 (FR1) for 1 hour or until receiving 60 reward outcomes in five sessions to avoid excessive ethanol intake. After acquiring the nose-poking behavior, the mice were trained on one session of random interval 30 (RI30) schedule for 30 minutes. The next day, mice were trained with two sessions of RI60 schedule for 30 minutes. In the FR1 schedule, one reward followed one nose-poke. In the RI30/60 schedule, there was a time-out interval averaging 30/60 seconds between two active nose-pokes. Figure 2B illustrates the operant chamber schedule for magazine training, FR1, and RI30/60 sessions. Inactive nose-pokes were not reinforced as a control.

Operant Conditioning with Ethanol Reward. We employed the same schedule as magazine training to the second session of the FR1 schedule. From the third session of FR1, we used $10 \mathrm{E}$ as the reward. FR1 sessions with $10 \mathrm{E}$ were for 30 minutes or until the mice had received 60 reward outcomes. After an additional two sessions of FR1 with 10E, the mice were conditioned for four sessions of fixed ratio 
2 (FR2) schedule for 30 minutes. The FR2 schedule resulted in a reward after two nose-pokes. Figure 4A illustrates the operant chamber schedule for FR2 sessions. Time spent in the magazine indicates how long mice stayed in the magazine (the place presenting the reward) per session duration.

Evaluation Test. For the evaluation session, the mice were given 1 hour of ad libitum access to tap water, 10E10S, or $10 \mathrm{E}$ and then underwent the extinction test sessions using the same training schedule (RI60 or FR2). The order of the neutral and devalued conditions was counterbalanced across mice. Extinction tests were 10 minutes in duration. Immediately after the extinction test, we performed RI60 or FR2 with reinforcement on the same day.

\section{Locomotor Activity Test}

The open-field test was conducted in chambers with sound attenuating cubicle (Med Associates). Mice were placed in the center of the field 30 minutes after the treatment (VEH or NHBA 0.03, 0.1, $0.3 \mathrm{mg} / \mathrm{kg}$, i.p.). An activity monitor (Med Associates) with infrared photobeam recorded the locomotor responses of the mice for 30 minutes.

\section{Three-Arm Choice Test Using the Y-Maze}

To examine the preference between water and $10 \mathrm{E}$, we employed the behavior test used in our previous research (Hong et al., 2019). Briefly, we put $7 \mathrm{ml}$ of tap water and $10 \mathrm{E}$ at the end of the Y-maze arm. Mice were under body weight restriction during both the pretests ( 1 day before the magazine training) and posttests ( 1 day after the last evaluation test), as illustrated in Fig. 2A. The length of each arm was $34 \mathrm{~cm}$, and we assigned start (no liquid), water, and 10E zones at the halfway point of each arm. Either vehicle or NHBA $(0.1 \mathrm{mg} / \mathrm{kg}$, i.p. $)$ was administered 30 minutes before the tests.

The treatment schedule was counterbalanced. We put mice in the start zone facing the wall, which allowed the mice to freely travel in the Y-maze. The video tracking system, EthoVision XT software (Noldus, Wageningen, Netherlands), recorded the zone entries, time spent in each zone, and total distance traveled for 15 minutes.

\section{Statistical Analysis}

All data are represented as mean \pm S.E.M. and were analyzed by unpaired/paired two-tailed Student's $t$ tests, one-way ANOVA/repeated measures one-way ANOVA followed by Bonferroni's multiple comparisons tests, and two-way ANOVA/repeated measures ANOVA followed by Bonferroni's multiple comparisons tests using Prism 7.0 (GraphPad Software, San Diego, CA). $P<0.05$ was considered statistically significant.

\section{Results}

NHBA Inhibits Ethanol-Drinking Behaviors in C57BL/6J Mice. Previously, our group found that pharmacologic activation of $\mathrm{A}_{2 \mathrm{~A}} \mathrm{R}$ (CGS21680) reduces ethanol drinking behaviors in ENT1 knockout (KO) but not wild-type (WT) mice (Nam et al., 2013b). Additionally, ENT1 KO mice tend to highly prefer alcohol when compared with WT mice (Choi et al., 2004). Thus, we developed a high ethanol drinking model to examine the effects of the novel adenosine analog NHBA, which activates $A_{2 A} R$, on ethanol drinking (Fig. 1A). After 26 days of ethanol drinking (3\% for 4 days $\rightarrow 6 \%$ for 4 days $\rightarrow 10 \%$ ethanol for 18 days), we found that around $60.5 \%$ of total mice (23 mice among total 38 mice) showed high ethanol drinking behaviors (Fig. 1B). During the $10 \%$ ethanol (10E) drinking, the mice exhibiting high ethanol drinking showed a higher preference (Fig. $1 \mathrm{C}, \mathrm{F}_{1,50}=341.6, P<0.001$ ) and consumption (Fig. 1D, $\mathrm{F}_{1,50}=157.1, P<0.001$ ) of ethanol rather than water ( $n=23$ /group).

Before examining the effects of NHBA on ethanol drinking, we performed the locomotor activity test to select an NHBA dose that does not affect locomotor activity. Systemic injection (i.p.) of NHBA $0.03(P=0.956)$ and $0.1 \mathrm{mg} / \mathrm{kg}(P=0.868)$ did not alter locomotion, whereas NHBA $0.3 \mathrm{mg} / \mathrm{kg}(P=0.001)$ reduced locomotor activities in the open-field test (Fig. 1E, $\mathrm{F}_{3,18}=8.01, P=0.001 ; n=7 / \mathrm{VEH}$ group, $n=5 / \mathrm{NHBA}$ groups); so we used NHBA $0.1 \mathrm{mg} / \mathrm{kg}$ for the ethanol drinking test.

Using ethanol-preferring mice, we investigated whether NHBA contributes to ethanol drinking. Before the treatment, the mice showed no differences in ethanol preference (Fig. $1 \mathrm{~F}$, $t=0.291, P=0.776$ ), consumption (Fig. 1G, $t=0.814, P=$ 0.433 ), nor total liquid consumption (Fig. $1 \mathrm{H}, t=0.402, P=$ 0.695 between VEH and NHBA treatment groups. NHBA $(0.1 \mathrm{mg} / \mathrm{kg}$, i.p.; treated at 18:00 hours and collected data at 21: 00 hours; $n=7 / \mathrm{VEH}$ group, $n=6 / \mathrm{NHBA}$ group) dampened ethanol preference (Fig. 1F, $t=3.058, P=0.011$ ) and consumption (Fig. $1 \mathrm{G}, t=2.492, P=0.030$ ), but NHBA did not alter total liquid consumption (Fig. $1 \mathrm{H}, t=0.990, P=$ 0.344). Therefore, NHBA may dampen ethanol consumption and preference in ethanol-preferring mice without altering locomotion.

NHBA Dampens Ethanol Preference after Operant Conditioning in C57BL/6J Mice. We next asked whether NHBA decreases operant conditioning-induced ethanol preference (Fig. 2A). We employed an RI schedule that facilitates maintaining conditioned reward values after FR1 training in the operant chamber (Yin and Knowlton, 2006; Gęsiarz and Crockett, 2015). In a different cohort from the ethanol drinking experiments, we examined the reinforcing effect of an ethanol-containing reward (10\% ethanol and 10\% sucrose, $10 \mathrm{E} 10 \mathrm{~S})$ after the acquisition of a nose-poking behavior in the FR1 schedule (Fig. 2B). In operant conditioning, the nosepoking behaviors were increased compared with the first FR1 session (Fig. 2C, $\mathrm{F}_{7,140}=11.19, P<0.001$ between session variations; from third FR1 to last training sessions, $P<0.001$; $n=11$ ), suggesting that the mice were conditioned to seek an ethanol-containing reward.

Then, using the Y-maze, we examined differences in ethanol preference between before and after operant conditioning. Heat maps displayed mice tracking on the Y-maze (Fig. 2D). Before conditioning with the ethanol-containing reward, neither the mice treated with VEH nor the NHBA mice $(0.1 \mathrm{mg} / \mathrm{kg}$, i.p.) showed significant changes in zone entries (Fig. $2 \mathrm{E}$; VEH group, $t=1.106, P=0.311, n=7$; NHBA group, $t=0.937, P=0.376, n=9$ ) or time spent in each zone (Fig. $2 \mathrm{~F}$, VEH group, $t=1.818, P=0.119, n=7$; NHBA group, $t=$ 2.097, $P=0.069, n=9$ ).

Interestingly, after operant conditioning, the VEH group exhibited higher zone entries (Fig. $2 \mathrm{E}, t=4.317, P=0.004$ ) and time spent (Fig. $2 \mathrm{~F}, t=5.556, P=0.001$ ) in the $10 \mathrm{E}$ zone compared with those in the water zone. However, NHBA treatment resulted in no significant differences in zone entries (Fig. $2 \mathrm{E}, t=1.047, P=0.330$ ) nor time spent (Fig. $2 \mathrm{~F}, t=$ 2.190, $P=0.065)$ between the water and $10 \mathrm{E}$ zones $(n=$ 8/group) without changing locomotor activity (Fig. $2 \mathrm{G}, t=0.567$, $P=0.579, n=8$ /group).

These results imply that operant conditioning with ethanolcontaining reward may increase ethanol preference and 
A

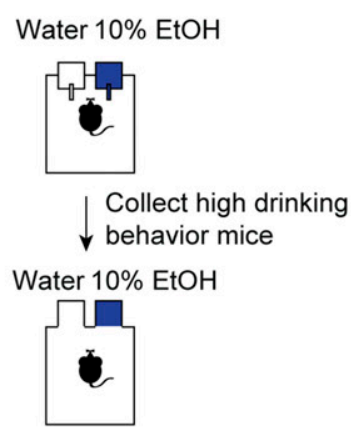

E

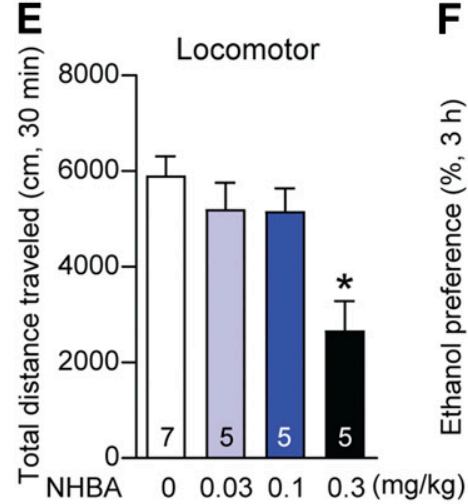

B

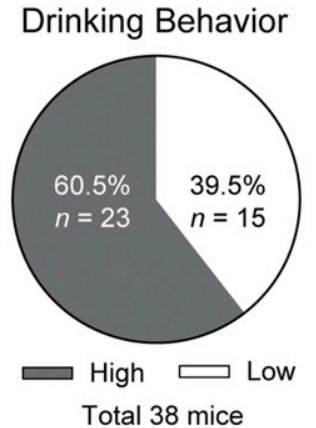

F

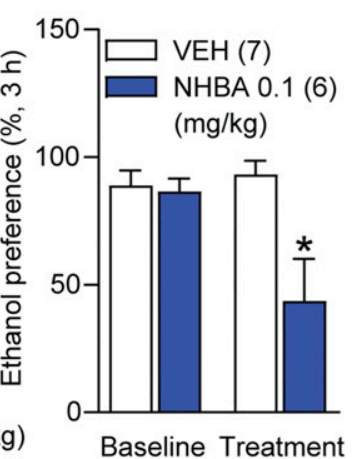

C

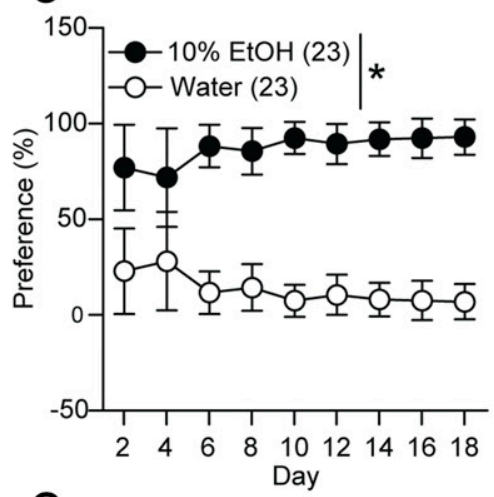

G

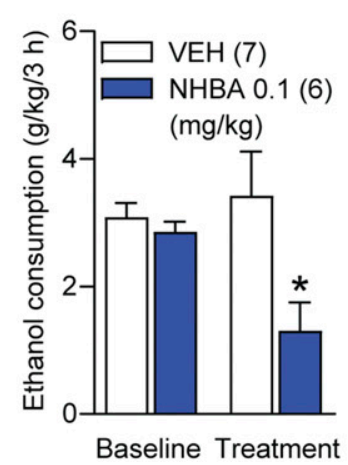

D

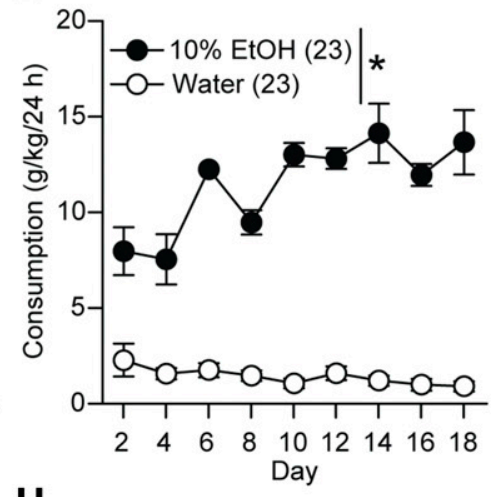

H

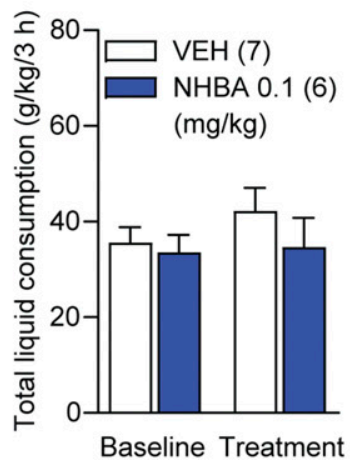

Fig. 1. Effects of NHBA on 10\% ethanol drinking in C57BL/6J mice. (A) Experimental scheme. (B) The ratio of mice showing high ethanol drinking behavior. (C) Ethanol preference and (D) ethanol consumption during $10 \%$ ethanol drinking ( $n=23$ /group). (E) Total distance traveled for 30 minutes in mice treated with NHBA $(0.03,0.1,0.3 \mathrm{mg} / \mathrm{kg}$; i.p.):- $n=7 / \mathrm{VEH}$ group, $n=5 / \mathrm{NHBA}$ groups. (F) Ethanol preference, $(\mathrm{G})$ consumption, and $(\mathrm{H})$ total liquid consumption in mice treated with NHBA $0.1 \mathrm{mg} / \mathrm{kg}$ (i.p.) for 3-hour drinking: $n=7 / \mathrm{VEH}$ group, $n=6 / \mathrm{NHBA}$ group. Data represented as mean \pm S.E.M. $* P<0.05$, (C and D) comparing water group, and (E-H) comparing vehicle treatment group. (C and D) Two-way repeated measures ANOVA followed by Bonferroni's multiple comparisons test, (E) one-way ANOVA followed by Bonferroni's multiple comparisons test, and (F-H) unpaired Student's $t$ test.

NHBA may reduce this operant conditioning-induced ethanol preference without changing exploration activity.

NHBA Dampens Operant Conditioning-Induced Revaluation toward Ethanol-Containing Reward in C57BL/6J Mice. Next, we employed the evaluation test, which is composed of an extinction test after presentation of unlimited water and $10 \mathrm{E}$ in the home cage for 1 hour, to investigate whether NHBA contributes to reward evaluation (Fig. 3, A and B). We presented water for the neutral state (as the control state) and 10E10S for the devalued state for 1 hour in mice home cages.

As expected, NHBA ( $0.1 \mathrm{mg} / \mathrm{kg}$, i.p. ) significantly decreased nose-poking behavior compared with the $\mathrm{VEH}$ group in the neutral state (Fig. 3C, $\mathrm{F}_{2,12}=0.027, P=0.018, n=5$ /group). Additionally, dampening nose-poking behaviors by NHBA $(0.1 \mathrm{mg} / \mathrm{kg}$, i.p.) treatment displayed no significant difference of nose-pokes between water and 10E10S (Fig. 3D, $t=1.829$, $P=0.141, n=5$ ). The VEH (Fig. $3 \mathrm{D}, t=4.570, P=0.010, n=$ $5 /$ group $)$ and NHBA $(0.03 \mathrm{mg} / \mathrm{kg}$, i.p.; Fig. $3 \mathrm{D}, t=3.184, P=$ $0.033, n=5$ ) groups showed significant changes of nosepoking between water and 10E10S presentation states.

These results suggest that NHBA at a $0.1 \mathrm{mg} / \mathrm{kg}$ dose decreases the value of the ethanol-containing reward outcome, similar to $10 \mathrm{E} 10 \mathrm{~S}$ presentation in the home cages. Based on the results for NHBA doses in ethanol drinking and evaluation tests, we used an NHBA dose $(0.1 \mathrm{mg} / \mathrm{kg}$, i.p.) for the next experiments. We examined whether ethanol alone presentation decreases the value of the operant conditioned ethanol- containing reward and how NHBA contributes to $10 \mathrm{E}$-induced reward devaluation.

Interestingly, ethanol presentation significantly reduced nose-pokes to the ethanol-containing reward in the $\mathrm{VEH}$ treatment group (Fig. $3 \mathrm{E}, t=4.940, P=0.002, n=8$ ), and NHBA treatment $(0.1 \mathrm{mg} / \mathrm{kg}$, i.p.) consistently devalued the ethanol-containing reward as the $10 \mathrm{E}$ presentation devalued it (Fig. 3E, $t=0.172, P=0.870, n=8$ ).

These results suggest that operant conditioning toward an ethanol-containing reward increases the value of ethanol whereas NHBA reduces the value of the ethanol-containing conditioned reward.

NHBA Decreases the Value of Ethanol Reward in C57BL/6J Mice. To further investigate whether NHBA alters the value of an ethanol reward outcome; we trained mice with $10 \mathrm{E} 10 \mathrm{~S}$ and then $10 \mathrm{E}$ only (Fig. 4, A and B). We used fixed ratio 2 (FR2) schedule to increase the reinforcing effect of ethanol reward and resilience to the extinction test after the stable acquisition of a nose-poking behavior in the FR1 schedule (Sangha et al., 2002; Wouda et al., 2010). After the transition to the $10 \mathrm{E}$ reward, the mice still showed stable seeking behavior (Fig. 4, C and D). In particular, the nosepoking behavior was increased during the conditioning with the $10 \mathrm{E}$ compared with the first FR1 session with the 10E10S reward (Fig. $4 \mathrm{C}, \mathrm{F}_{8,128}=2.382, P=0.020$ between session variations; in first FR2 session, $P=0.034$; in third FR2 session, $P=0.022$; in fourth FR2 session, $P<0.001$; $n=11$ group). 
A Pre

10E10S-Seeking Behavior

Post

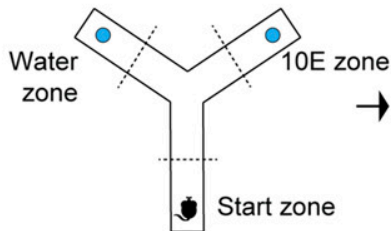

B

Operant conditioning with $10 \mathrm{E} 10 \mathrm{~S}$
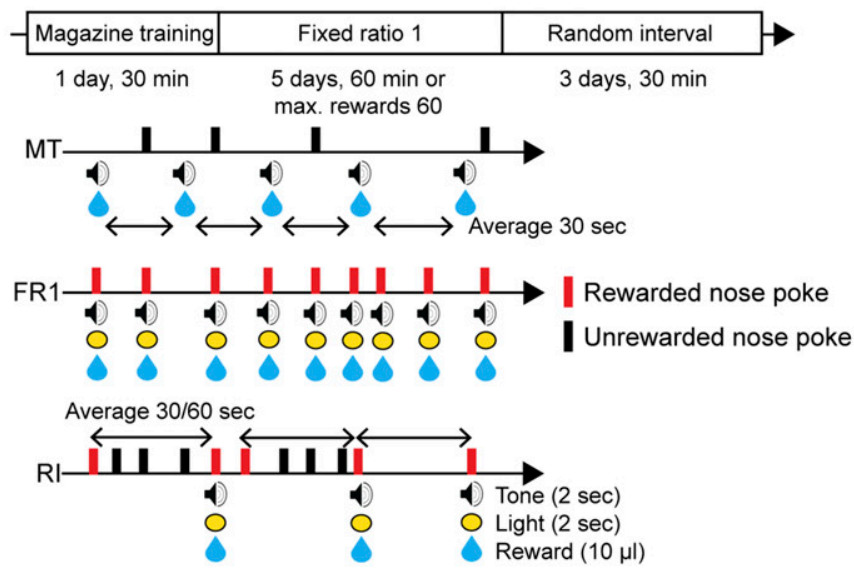

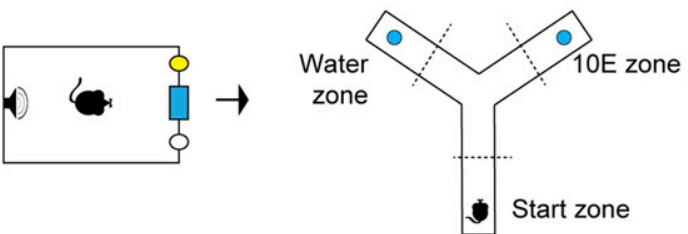

C

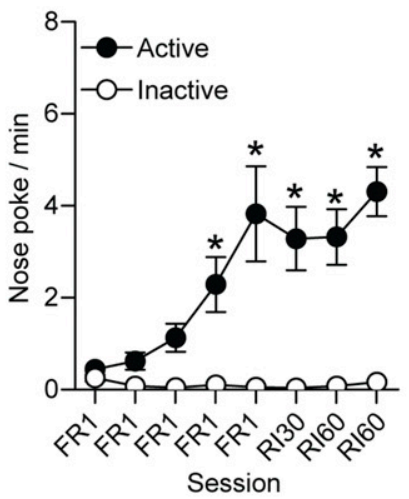

Session

D

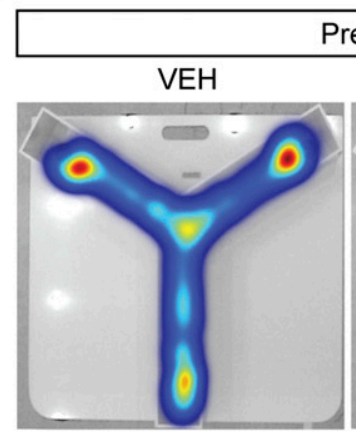

E

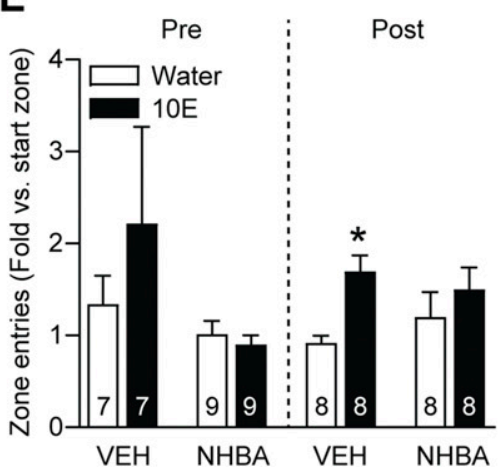

$\mathbf{F}$

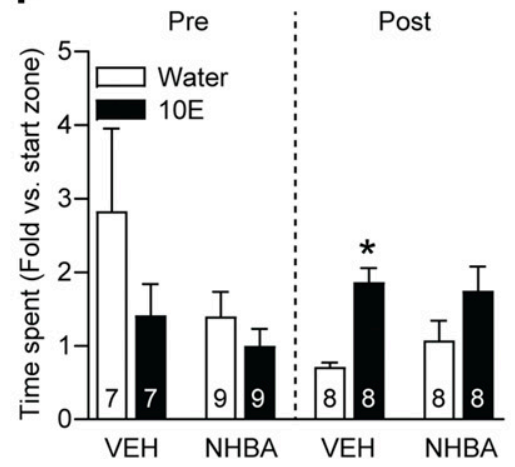

Post

VEH

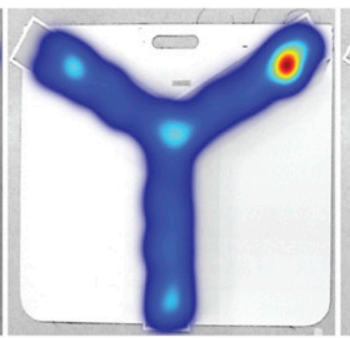

G

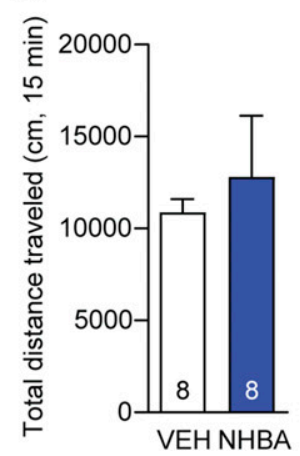

Fig. 2. Effects of NHBA on ethanol preference before and after training with ethanol-containing reward. (A and B) Experimental scheme. (C) Nosepoking behavior ( $n=11$ /group). (D) Heat map depicting mice tracking patterns for 15 minute on the Y-maze. (E) Zone entries and (F) time spent in each zone of the Y-maze ( $n=7 / \mathrm{VEH}+$ Pre, $n=9 / \mathrm{NHBA}+$ Pre, $n=8 /$ post groups). (G) Total distance traveled for 15 minutes among groups $(n=8 /$ groups). Data represented as mean \pm S.E.M. $* P<0.05$, (C) comparing the first FR1 session and (E and F) comparing the water zone of the same treatment group. (C) Two-way repeated measures ANOVA followed by Bonferroni's multiple comparisons test, (E and F) one-way repeated measures ANOVA followed by Bonferroni's multiple comparisons test, and (G) unpaired Student's $t$ test.

On the evaluation test, we presented water for the neutral state and $10 \mathrm{E}$ for the devalued state. As expected, NHBA $(0.1 \mathrm{mg} / \mathrm{kg}$, i.p.) decreased the nose-pokes compared with the
VEH group in the water presentation state (Fig. $4 \mathrm{E}, t=3.005$, $P=0.008, n=11 /$ group $)$. NHBA $(0.1 \mathrm{mg} / \mathrm{kg}$, i.p. $)$ also exhibited no significant difference between the water and $10 \mathrm{E}$ 
A

B

Operant conditioning with $10 \mathrm{E} 10 \mathrm{~S}$

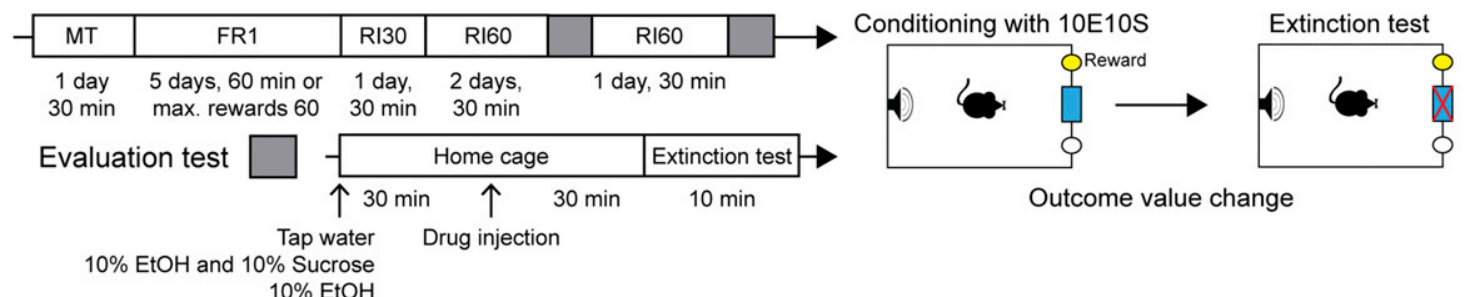

C

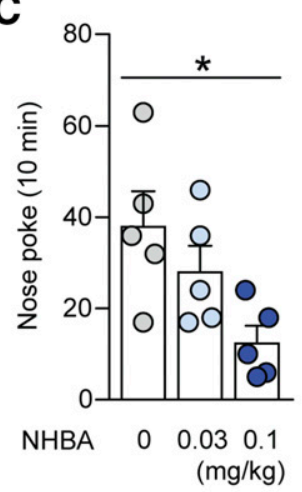

D

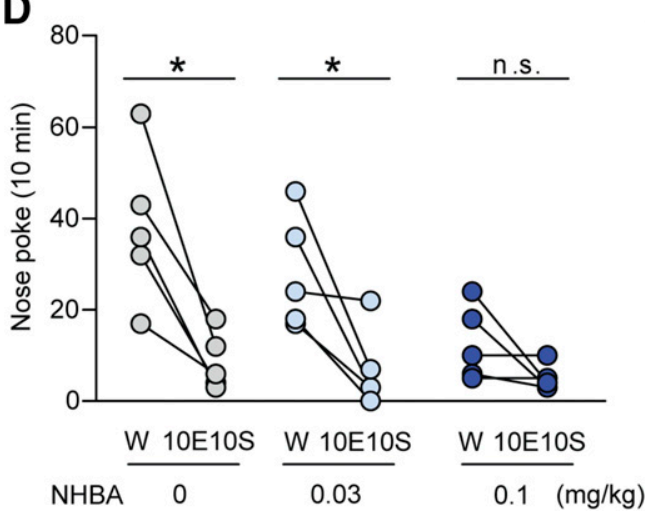

E

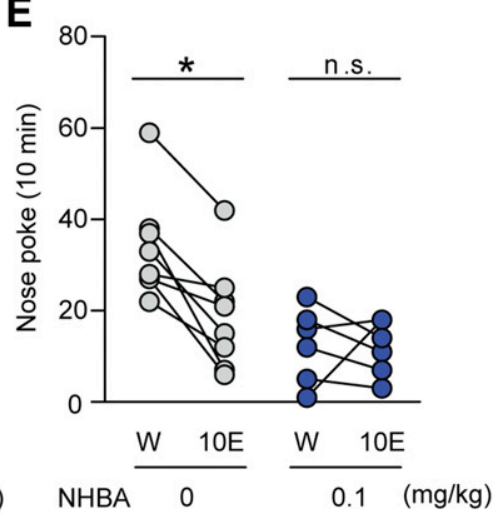

Fig. 3. Effects of NHBA on ethanol-containing reward-seeking in C57BL/6J mice. (A and B) Experimental scheme. (C) Effects of NHBA (0.03 and $0.1 \mathrm{mg} / \mathrm{kg}$, i.p.) on nose-poking behavior in water (W) presentation state. (D) Nose-poking changes between water (W) and $10 \%$ ethanol and $10 \%$ sucrose (10E10S) presentation states $(n=5 /$ group). (E) Effects of NHBA $(0.1 \mathrm{mg} / \mathrm{kg}$, i.p.) on nose-poking behavior in either water (W) or $10 \%$ ethanol (10E) presentation state $(n=8$ /group). Data represented as mean \pm S.E.M. $* P<0.05$, (C) comparing vehicle treatment group, (D and E) comparing each different state. (C) One-way ANOVA followed by Bonferroni's multiple comparisons test, and (D and E) paired Student's $t$ test.

presentation states (Fig. $4 \mathrm{~F}, t=1.219, P=0.258, n=11$ ), but the VEH group showed a significant difference between the two states (Fig. $4 \mathrm{~F}, t=2.403, P=0.043, n=11$ ). These results indicate that NHBA may dampen ethanol outcome's value in mice.

\section{Discussion}

Our study is the first to date to examine the effects of the active compound NHBA (in the rhizome of Gastrodia elata) on ethanol-related behaviors. Our findings highlight the importance of purinergic adenosine tone to the modulation of ethanol drinking and seeking behaviors. We found that dual ENT1 inhibition along with $\mathrm{A}_{2 \mathrm{~A}} \mathrm{R}$ activation reduced ethanol drinking behaviors in mice exhibiting high ethanol drinkingbehavior. Moreover, operant conditioning with an ethanolcontaining reward increased the preference of the ethanol zone and the value of ethanol. However, the novel adenosine analog NHBA dampened the ethanol-containing reward and ethanol reward seeking behaviors.

Because initiating ethanol intake in the operant conditioning paradigm is difficult in mice, chronic ethanol adaptation, sucrose fading, or water/food restriction has been widely used to facilitate operant conditioning (Corbit et al., 2012; Ron and Barak, 2016; Blegen et al., 2018). In this study, we found that mice under body weight restriction did not preferably approach and stay in the ethanol zone before the operant conditioning, whereas operant conditioning with ethanolcontaining reward increased the preference toward ethanol zone in the Y-maze. This is consistent with our previous study, which revealed that operant conditioning with an ethanol-containing reward increases the preference for an ethanol-containing solution zone (Hong et al., 2019). Additionally, we found that a single treatment of NHBA reduced this increase in ethanol zone preference, which suggests that ENT1 and $\mathrm{A}_{2 \mathrm{~A}} \mathrm{R}$ are associated with the development of ethanol reward behaviors.

We assessed the valence of the reward (ethanol-containing and ethanol rewards) in the evaluation test by presenting water (for the neutral state), ethanol-containing (10E10S), or ethanol solution itself (10E) (for the devalued state) before the extinction test. This method was modified from methods to examine both goal-directed and habitual seeking behaviors (Corbit et al., 2012; Gremel and Costa, 2013). Our findings suggest a rapid transition from ethanol-containing reward seeking to ethanol-only seeking behaviors during the FR1 schedule. However, further study is needed to identify the role of NHBA in goal-directed and habitual ethanol seeking behaviors.

The voluntary two-bottle choice assay used to evaluate ethanol consumption and general avidity to ethanol induces incremental escalation of consumption, which is thought to reprise many clinical hallmarks of alcoholism (Leeman et al., 2010; Bosse et al., 2019). Ethanol exposure is associated with altered expression and activity of ENT1 and $\mathrm{A}_{2 \mathrm{~A}} \mathrm{R}$ (Nagy et al., 1990; Choi et al., 2004). Mice without ENT1 display reduced hypnotic and ataxic behaviors to acute ethanol administration as well as increased voluntary ethanol consumption compared with WT mice (Choi et al., 2004). Because $\mathrm{A}_{2 \mathrm{~A}} \mathrm{R}$ has the lowest binding affinity for adenosine, the reduction of adenosine levels by ENT1 ablation mainly affects $\mathrm{A}_{2 \mathrm{~A}} \mathrm{R}$ and its subsequent signaling cascade, which may contribute to excessive 
A Operant conditioning

Conditioning with 10E10S Conditioning with $10 \mathrm{E}$

\begin{tabular}{|c|c|c|c|c|c|c|c|}
\hline \hline MT & FR1 & FR1 & FR2 & FR2 & FR2 & FR2 \\
1 day 3 days, 60 min or 2 days, 30 min or 4 days & 1 day 1 day 1 day \\
30 min max. rewards 60 max. rewards 6030 min & 30 min 30 min 30 min \\
Evaluation test
\end{tabular}

B

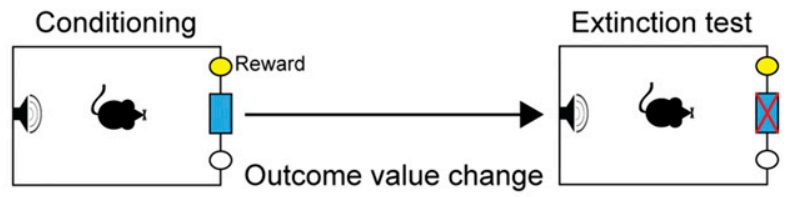

D

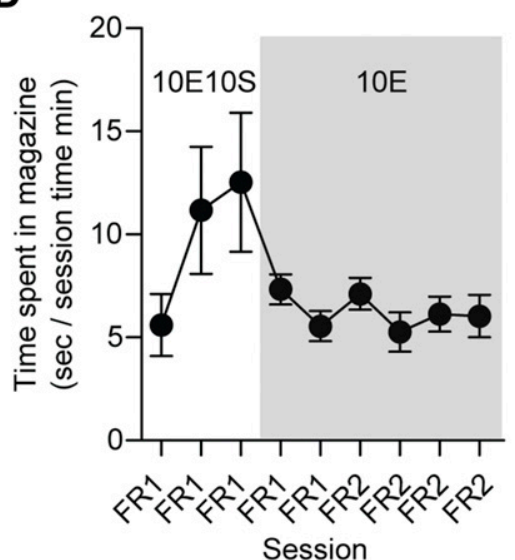

E

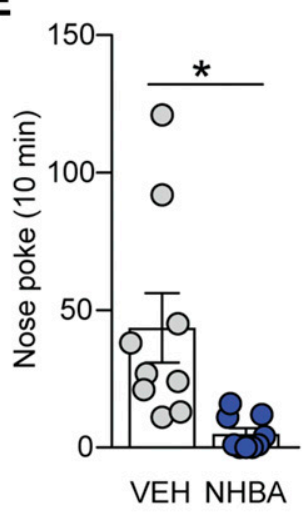

C

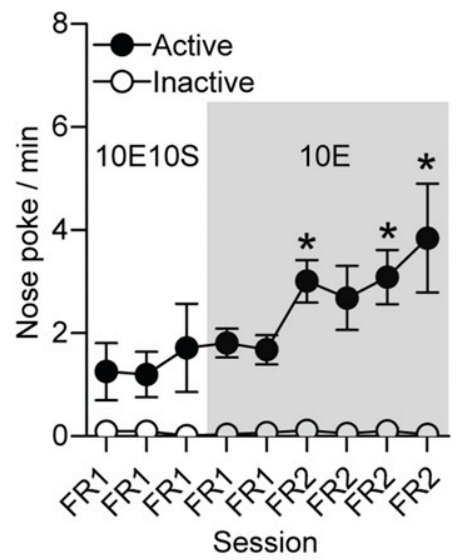

F

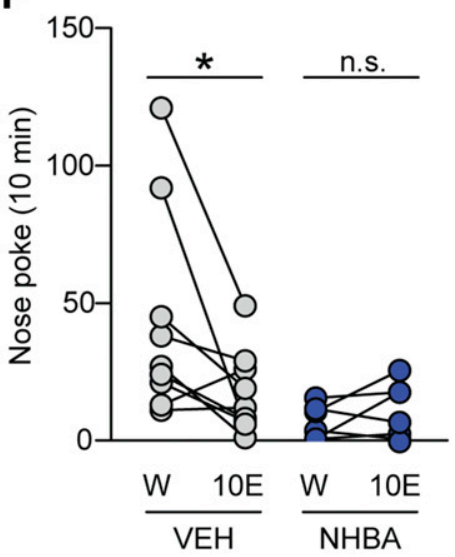

Fig. 4. Effects of NHBA on ethanol reward-seeking in C57BL/6J mice. (A and B) Experimental scheme. (C) Nose-poking behavior and (D) time spent in the magazine. (E) Effects of NHBA $(0.1 \mathrm{mg} / \mathrm{kg}$, i.p.) on nose-poking behavior in water (W) presentation state. (F) Nose-poking changes between water (W) and $10 \%$ ethanol $(10 \mathrm{E})$ presentation states. Data represented as mean \pm S.E.M., $n=11$ /groups. $* P<0.05$, (C) comparing the first FR1 session, (E) comparing vehicle treatment group, (F) comparing each different state. (C) Two-way repeated measures ANOVA by Bonferroni's multiple comparisons test, (E) unpaired Student's $t$ test, (F) paired Student's $t$ test.

ethanol consumption in ENT1 KO mice (Nam et al., 2011, 2013a).

Interestingly, $\mathrm{A}_{2 \mathrm{~A}} \mathrm{R}$ inhibition augments voluntary ethanol intake and preference in WT mice, whereas $\mathrm{A}_{2 \mathrm{~A}} \mathrm{R}$ activation dampens ethanol drinking behaviors only in ENT1 KO but not in WT mice (Nam et al., 2013b). The present study corroborated that NHBA, an ENT1 antagonist and $\mathrm{A}_{2 \mathrm{~A}} \mathrm{R}$ agonist, would dampen ethanol drinking behavior in WT mice displaying high ethanol consumption during voluntary drinking. This implies that the dual-function toward ENT1 and $\mathrm{A}_{2 \mathrm{~A}} \mathrm{R}$ may be a more promising therapeutic strategy to treat AUD rather than an isolated $\mathrm{A}_{2 \mathrm{~A}} \mathrm{R}$ targeting ligand.

The limited number of therapeutic options available for the treatment of AUD is troubling. Unfortunately, dangerous alcohol consumption is increasing in the United States (Kranzler and Soyka, 2018). Modulation of adenosine signaling by NHBA represents a promising alternative to suppress ethanol consumption and preference. Interestingly, our findings suggest that NHBA might be useful in reducing the valuation of rewarding substances, suggesting that this compound may help reduce drug-seeking or relapse to drugseeking behavior.

\section{Acknowledgments}

We thank Matthew Baker and DeAnn Frederixon for their proofreading and editing of the manuscript.

Competing Interests: Dr. D.-S.C. is a scientific advisory board member to Peptron Inc., which had no role in preparation, review, or approval of the manuscript; nor decision to submit the manuscript for publication. All the other authors declare no biomedical financial interests or potential conflicts of interest.

\section{Authorship Contributions}

Participated in research design: Hong, Peyton, Choi.

Conducted experiments: Hong, Peyton.

Contributed new reagents or analytic tools: Chern.

Performed data analysis: Hong, Peyton.

Wrote or contributed to the writing of the manuscript: Hong, Peyton, Chern, Choi. 


\section{References}

Blegen MB, da Silva E Silva D, Bock R, Morisot N, Ron D, and Alvarez VA (2018) Alcohol operant self-administration: investigating how alcohol-seeking behaviors predict drinking in mice using two operant approaches. Alcohol 67:23-36.

Bosse KE, Ghoddoussi F, Eapen AT, Charlton JL, Susick LL, Desai K, Berkowitz BA Perrine SA, and Conti AC (2019) Calcium/calmodulin-stimulated adenylyl cyclases 1 and 8 regulate reward-related brain activity and ethanol consumption. Brain Imaging Behav 13:396-407.

Chen WC, Lai YS, Lin SH, Lu KH, Lin YE, Panyod S, Ho CT, and Sheen LY (2016) Anti-depressant effects of Gastrodia elata Blume and its compounds gastrodin and 4-hydroxybenzyl alcohol, via the monoaminergic system and neuronal cytoskeletal remodeling. J Ethnopharmacol 182:190-199.

Choi DS, Cascini MG, Mailliard W, Young H, Paredes P, McMahon T, Diamond I, Bonci A, and Messing RO (2004) The type 1 equilibrative nucleoside transporter regulates ethanol intoxication and preference. Nat Neurosci 7:855-861.

Corbit LH, Nie H, and Janak PH (2012) Habitual alcohol seeking: time course and the contribution of subregions of the dorsal striatum. Biol Psychiatry 72:389-395.

De Witte P, Littleton J, Parot P, and Koob G (2005) Neuroprotective and abstinencepromoting effects of acamprosate: elucidating the mechanism of action. CNS Drugs 19:517-537.

Ferré S, Bonaventura J, Zhu W, Hatcher-Solis C, Taura J, Quiroz C, Cai NS, Moreno E, Casadó-Anguera V, Kravitz AV, et al. (2018) Essential control of the function of the striatopallidal neuron by pre-coupled complexes of adenosine $\mathrm{A}_{2 \mathrm{~A}}$-dopamine $\mathrm{D}_{2}$ receptor heterotetramers and adenylyl cyclase. Front Pharmacol 9:243.

Gęsiarz F and Crockett MJ (2015) Goal-directed, habitual and Pavlovian prosocial behavior. Front Behav Neurosci 9:135.

Gremel CM and Costa RM (2013) Orbitofrontal and striatal circuits dynamically encode the shift between goal-directed and habitual actions. Nat Commun 4:2264

Hong SI, Kang S, Chen JF, and Choi DS (2019) Indirect medium spiny neurons in the dorsomedial striatum regulate ethanol-containing conditioned reward seeking. $J$ Neurosci [published ahead of print] DOI: 10.1523/JNEUROSCI.0876-19.2019.

Huang NK, Chern Y, Fang JM, Lin CI, Chen WP, and Lin YL (2007) Neuroprotective principles from Gastrodia elata. J Nat Prod 70:571-574.

Huang NK, Lin JH, Lin JT, Lin CI, Liu EM, Lin CJ, Chen WP, Shen YC, Chen HM, Chen JB, et al. (2011) A new drug design targeting the adenosinergic system for Huntington's disease. PLoS One 6:e20934.

Jørgensen CH, Pedersen B, and Tønnesen H (2011) The efficacy of disulfiram for the treatment of alcohol use disorder. Alcohol Clin Exp Res 35:1749-1758.

Juarez B, Morel C, Ku SM, Liu Y, Zhang H, Montgomery S, Gregoire H, Ribeiro E, Crumiller M, Roman-Ortiz C, et al. (2017) Midbrain circuit regulation of individual alcohol drinking behaviors in mice [published correction appears in Nat Commun (2018) 9:653]. Nat Commun 8:2220.

Jung JW, Yoon BH, Oh HR, Ahn JH, Kim SY, Park SY, and Ryu JH (2006) Anxiolytic-like effects of Gastrodia elata and its phenolic constituents in mice. Biol Pharm Bull 29:261-265.

Kao YH, Lin MS, Chen CM, Wu YR, Chen HM, Lai HL, Chern Y, and Lin CJ (2017) Targeting ENT1 and adenosine tone for the treatment of Huntington's disease. Hum Mol Genet 26:467-478.

Kranzler HR and Soyka M (2018) Diagnosis and pharmacotherapy of alcohol use disorder: a review. JAMA 320:815-824.
Leeman RF, Heilig M, Cunningham CL, Stephens DN, Duka T, and O'Malley SS (2010) Ethanol consumption: how should we measure it? Achieving consilience between human and animal phenotypes. Addict Biol 15:109-124.

Li Y, He Y, Chen M, Pu Z, Chen L, Li P, Li B, Li H, Huang Z-L, Li Z, et al. (2016) Optogenetic activation of adenosine A2A receptor signaling in the dorsomedial striatopallidal neurons suppresses goal-directed behavior. Neuropsychopharmacology 41:1003-1013.

Nagy LE, Diamond I, Casso DJ, Franklin C, and Gordon AS (1990) Ethanol increase extracellular adenosine by inhibiting adenosine uptake via the nucleoside transporter. J Biol Chem 265:1946-1951.

Nam HW, Bruner RC, and Choi DS (2013a) Adenosine signaling in striatal circuits and alcohol use disorders. Mol Cells 36:195-202.

Nam HW, Hinton DJ, Kang NY, Kim T, Lee MR, Oliveros A, Adams C, Ruby CL, and Choi DS (2013b) Adenosine transporter ENT1 regulates the acquisition of goal-directed behavior and ethanol drinking through A2A receptor in the dorsomedial striatum. $J$ Neurosci 33:4329-4338.

Nam HW, Lee MR, Zhu Y, Wu J, Hinton DJ, Choi S, Kim T, Hammack N, Yin JC, and Choi DS (2011) Type 1 equilibrative nucleoside transporter regulates ethanol drinking through accumbal $N$-methyl-D-aspartate receptor signaling. Biol Psychiatry 69:1043-1051.

Oliveros A and Choi D-S (2017) Repurposing tigecycline for the treatment of alcohol use disorder. Alcohol Clin Exp Res 41:497-500.

Ron D and Barak S (2016) Molecular mechanisms underlying alcohol-drinking behaviours. Nat Rev Neurosci 17:576-591.

Ruby CL, Adams CA, Knight EJ, Nam HW, and Choi D-S (2010) An essential role for adenosine signaling in alcohol abuse. Curr Drug Abuse Rev 3:163-174.

Sachdeva S and Gupta M (2013) Adenosine and its receptors as therapeutic targets: an overview. Saudi Pharm J 21:245-253.

Sangha S, McComb C, Scheibenstock A, Johannes C, and Lukowiak K (2002) The effects of continuous versus partial reinforcement schedules on associative learning, memory and extinction in Lymnaea stagnalis. J Exp Biol 205: 1171-1178.

Wackernah RC, Minnick MJ, and Clapp P (2014) Alcohol use disorder: pathophysiology, effects, and pharmacologic options for treatment. Subst Abuse Rehabil $\mathbf{5}$ $1-12$

Wouda JA, Diergaarde L, Riga D, van Mourik Y, Schoffelmeer AN, and De Vries TJ (2010) Disruption of long-term alcohol-related memory reconsolidation: role of $\beta$-adrenoceptors and NMDA receptors. Front Behav Neurosci 4:179.

Yin $\mathrm{HH}$ and Knowlton BJ (2006) The role of the basal ganglia in habit formation. Nat Rev Neurosci 7:464-476.

Zhang Y, Li M, Kang RX, Shi JG, Liu GT, and Zhang JJ (2012) NHBA isolated from Gastrodia elata exerts sedative and hypnotic effects in sodium pentobarbitaltreated mice. Pharmacol Biochem Behav 102:450-457.

Address correspondence to: Dr. Doo-Sup Choi, Department of Molecular Pharmacology and Experimental Therapeutics, Mayo Clinic College of Medicine, 200 First Street SW, Rochester, MN 55905. E-mail: choids@ mayo.edu 